\title{
Influencia de la intervención humana en procesos modernos de manufactura: un enfoque de simulación de procesos centrado en el factor humano
}

\section{Influence of human intervention on the modern manufacturing processes: an approach to process simulation focused on the human factor}

Carmen Madriz ${ }^{1}$, Magaly Sánchez², Olga Sánchez ${ }^{3}$, Juan B. Hernández-Granados ${ }^{4}$

Fecha de recepción: 4 de octubre de 2020 Fecha de aprobación: 18 de enero de 2021

Madriz, C; Sánchez, M; Sánchez, O; Hernández-Granados, J.B. Influencia de la intervención humana en procesos modernos de manufactura: un enfoque de simulación de procesos centrado en el factor humano. Tecnología en Marcha.

Vol. 35-1. Enero-Marzo 2022. Pág 3-13.

doi https://doi.org/10.18845/tm.v35i1.5358

1 Profesora de la Escuela de Ingeniería en Producción Industrial del Instituto Tecnológico de Costa Rica (TEC) e investigadora del Laboratorio de Ergonomía del TEC. Costa Rica. Correo electrónico: cmadriz@tec.ac.cr

2 Profesora de la Escuela de Ingeniería en Producción Industrial del Instituto Tecnológico de Costa Rica (TEC) e investigadora del Laboratorio de Ergonomía del TEC.

3 Profesora de la Escuela de Ingeniería en Diseño Industrial del Instituto Tecnológico de Costa Rica (TEC) e investigadora del Laboratorio de Ergonomía del TEC. Costa Rica.

4 Profesor de la Escuela de Ingeniería en Producción Industrial del Instituto Tecnológico de Costa Rica (TEC) e investigadora del Laboratorio de Manufactura del TEC. Costa Rica. 


\section{Palabras clave}

Error humano; simulación; fiabilidad humana.

\section{Resumen}

El presente proyecto se basa en la modelación de la relación hombre-máquina centrado en el factor humano y en los errores que no necesariamente llevan a fatalidades, pero si a pérdidas de productividad. Este caso de estudio se basa en un proceso automatizado típico en una empresa metalmecánica. Error del set-ut, especificación y medidas tienen una probabilidad de ocurrencia de 0.1, 0.05 y 0.2 respectivamente. Dependiendo de la etapa en el proceso así será el efecto del error humano ya sea en el tiempo de terminación o en la cantidad de unidades producidas buenas. La simulación de proceso es usada en la evaluación del impacto del error humano en los diferentes puntos del proceso. La técnica Technique for Human Error Rate Prediction (THERP) es usada para la estimación del error humano. Los programas de entrenamiento y estandarización de procesos son herramientas claves para la prevención de estos errores.

\section{Keywords}

Human error; simulation; human reliability.

\section{Abstract}

This project is based on modeling the human-machine relationship. It focuses on the human factor and on errors that do not necessarily lead to fatalities, but to productivity losses. The case study is based on a typical automated process in a metalworking company. Errors in set-up, specification and measurement have a probability of $0.1,0.05$ and 0.2 respectively. Depending on the process stage, the effect of human error will be either on completion time or in the number of correctly produced units. Process simulation was used in the assessment of the impact of human error at different points in the process. The Technique for Human Error Rate Prediction (THERP) was used for the estimation of human error. Training programs and process standardization are essential tools in preventing these mistakes.

\section{Introducción}

Tradicionalmente la atención en los errores humanos se ha dado a causa de grandes fatalidades, Madhu y otros, 2006 mencionan como grandes sucesos relacionados con errores humanos el sucedido en los almacenes de Petróleo en Buncefield, UK en el 2005 y la explosión de los almacenes de fertilizantes al oeste de Texas en el 2013 entre otros [1]. Sin dejar de restarle importancia a estos eventos existen los eventos diarios y silencios relacionados en el error humano que ocurren continuamente en el piso de la planta de producción. El rol humano en los sistemas modernos de manufactura ha cambiado significativamente en los últimos años, especialmente con la inserción de las tecnologías de control numérico (CNC). Los errores asignables a las máquinas son cada vez menores por el nivel tecnológico involucrado pero el nivel de error humano decrece lentamente en comparación con los errores asignados a las máquinas. Wiegmann, Shappell [2]. Kidam y otros, [3], establece que los errores de los operarios tales como desconcentraciones por periodos cortos, pérdida de memoria temporal, descuidos, no seguimiento de protocolos en el proceso son a menudo la causa de que el equipo o maquinaria de planta se encuentre inactiva o en modo de falla. 
El nivel de preparación del personal y su nivel técnico se han incrementado con el advenimiento de los nuevos sistemas de manufactura. Nuevas empresas que llegan al país traen consigo nuevos sistemas productivos con dedicaciones en mano de obra multifuncionales. Por ejemplo, la filosofía de la manufactura Lean establece que se debe tener grupos de trabajadores más colaborativos y multifuncionales, requiriendo mayores habilidades y conocimiento así como experiencia de estos, Solis y Madriz [4]. Zhang y Sharifi [5] establecen que estas características de los nuevos empleados son críticas para la agilidad de las organizaciones.

La International Ergonomics Association (IEA) y la International Commission on Occupational Health $(\mathrm{ICOH})$ [6] desde el 2008 han definido el error humano como "una decisión o comportamiento humano inapropiado o no deseable que produce, o tiene el potencial para producir, una reducción en la efectividad, salud y seguridad del trabajador, o una interrupción en el comportamiento del sistema"

Los desarrollos de tecnologías como las celdas de manufactura basada en el control numérico por medio del computador (CNC) nuevamente requieren de operarios más calificados para lograr una interacción exitosa del hombre y la máquina. El sistema tradicional de estudio del trabajo nos permite medir la eficiencia y productividad en trabajos de intensa mano de obra, sin embargo, conforme el nivel de manufactura se hace más flexible y automatizado el rol del ser humano es más difícil de evaluar por los sistemas tradicionales. Zhang y otros, [7].

Muchos de estos sistemas se basan en el conocimiento, donde existe gran cantidad de diversos conocimientos que se emplean en el desempeño de una tarea dada, y es aquí donde la relación hombre- máquina toma gran importancia en cada proceso del sistema.

Los errores humanos en este tipo de relación de sistemas hombre máquina se convierten en factores críticos para la eficiencia de los sistemas productivos o de servicios. Sanders y McCormick [7], define que el error humano son decisiones inapropiadas y no deseadas que interfieren en la efectividad, rendimiento y seguridad del trabajo.

Ya desde los años 50 el análisis de la confiabilidad en los sistemas no solo incluye el análisis de los equipos y máquinas sino también el factor humano. Sin embargo, el control de procesos se ha basado en las fallas de máquina y el control sobre las fallas humanas es casi nulo en estos sistemas. La cuantificación del error humano es en muchos casos una labor difícil, teniéndose que recurrir a metodologías propuestas de cuantificación.

Existen diferentes metodologías estandarizadas para la determinación del error humano, entre ellas se encuentra el método THERP (Technique for Human Error Rate Prediction) desarrollada por Swain y Guttman (1983) [9]. Esta es la técnica más conocida y la más representativa desde la perspectiva de la fiabilidad humana; utilizada para la obtención de datos sobre fiabilidad en los estudios de evaluación de probabilidades de riesgos. Su función es predecir las probabilidades de error humano y evaluar el deterioro de un sistema individuo-máquina causado por los errores humanos, por los procedimientos o las prácticas de ejecución, así como por las otras características del sistema o de la persona que influyen en el comportamiento del mismo. Cárdenas, M. [10].

Para el análisis de las operaciones humanas requeridas, se desarrolla el análisis detallado de la tarea. Se identifican los pasos necesarios para el desarrollo y el comportamiento de la acción y se analizan los posibles errores que se puedan realizar. Las categorías de errores posibles según el THERP, son: errores de omisión (omisión de un paso de la tarea) y errores de comisión o acción. Estos últimos pueden ser de cuatro tipos: Error de selección (selección del control erróneo, elección de procedimientos de forma incorrecta), Error en la secuencia (acciones llevadas a cabo en orden incorrecto), Error temporal (acciones llevadas a cabo muy pronto / muy tarde) y Error cualitativo (acción realizada en mucho / en poco). Sureda, J. [11]. 
La simulación dinámica de los procesos se ha convertido en una herramienta poderosa para estimar la efectividad de los sistemas de manufactura con un enfoque tradicional hacia el funcionamiento de las máquinas, pero tomando en cuenta la interacción con el ser humano que permita valorar en forma más integral el funcionamiento de un sistema productivo. Zhang, Smith, [12] proponen que la simulación que incluye el factor humano o la simulación centrada en el ser humano considera a este como autor importante en el desarrollo exitoso de una labor productiva.

El ser humano es considerado en este modelo como un componente crítico, el cual impacta el rendimiento final del sistema, Laughery [13]. Por lo tanto, el modelo incluye un detallado espectro de la interacción del hombre y la máquina. En el caso de la máquina se caracteriza también en elementos como: ordenes de manufactura, tiempo de procesamiento de la máquina, sistema de distribución, horarios y controles.

Actualmente la simulación dinámica de eventos discretos se está dirigiendo al enfoque centrado en el ser humano. Zuelch [14] ha usado redes activas para establecer un modelo del proceso de decisión para trabajadores de manufactura mediante la simulación de los equipos y operaciones de mantenimiento. Robinson [15] estableció un modelo de simulación visual para tomar decisiones por una persona con el objetivo de evaluar el proceso de toma de decisiones. Zee [16] realizó una simulación orientada a los objetos para modelar métodos de manufactura incluyendo métodos de control. Zhang y Xie [17] han desarrollado recientemente simulaciones del impacto de la organización del trabajo en celdas de manufactura basado en el modelo centrado en el ser humano, donde en este caso se incorpora el factor de trabajo en equipo.

Sin lugar a duda el estudio del factor humano en el proceso de decisión en la relación hombremáquina, es clave para el mejoramiento de la productividad de los procesos, aún más cuando estas relaciones se dan en un ambiente de manufactura y tecnologías modernas, donde el nivel de decisión es más racional y las repercusiones de los errores humanos son más costosas tanto para el trabajador como para la empresa en términos de seguridad, calidad y costo.

\section{Materiales y métodos}

Este trabajo se realizó en una empresa dedicada al área de metalmecánica con tecnología de punta de control numérico, específicamente en el proceso de tornos primarios donde se da la forma y revisión de medidas a las piezas. Se realizaron diagramas de flujo, de operaciones y bimanuales para tipificar los movimientos y tareas que se realizan en las diferentes partes de la labor. El tamaño del lote analizado es de 5000 piezas y se utilizó adicionalmente el método de la observación por medio de muestreo. Por un tiempo de tres meses se efectúa la recolección de datos y se utilizan formatos diseñados, así como medios digitales (videos) para su posterior análisis e identificación y clasificación del error humano en las labores de la operación.

Para el modelaje de las operaciones se establecieron las variables de tiempos de proceso con su respectiva distribución de probabilidad, para esto se tomó una muestra de tiempos de 30 observaciones para cada operación. En la operación de torno se identificaron los errores humanos y se clasificaron con base a la norma NTP 620, (Sureda, [11] en errores de omisión y en errores de acción; éstos últimos se derivan en errores de selección, error de secuencia, error temporal y error cualitativo. A cada error se asignó un número de probabilidad basado en la metodología THERP, según la norma NTP 620 [18], para determinar las probabilidades de error en las diferentes tareas de la operación de torno. El flujo lógico del modelo se construyó en el software licenciado Arena 14.7. 
Se realizaron las pruebas de bondad de ajuste para la determinación del comportamiento de las variables aleatorias de tiempo de proceso utilizando el método Anderson-Darling con un nivel de significancia de 0.05. El número de réplicas de la simulación fue determinado con un 95\% de confianza y un 3\% de error en los datos, considerando como parámetro de referencia el porcentaje de utilización del sistema. Se realizaron dos modelaciones base una centrada en la situación actual que incluye todos los errores humanos identificados y la otra en la disminución del error humano mediante buenas prácticas de manufactura en dos puntos diferentes del proceso, y se comparan sus resultados mediante pruebas de hipótesis de medias con un 95\% de confianza y un 5\% de precisión. La validación del modelo se realiza por medio del criterio de experto en una reunión colaborativa tanto de los investigadores como de los expertos del sistema.

El modelo de simulación del proceso de torno automatizado considera los seis errores determinados y los procesos productivos que se asocian a los trabajadores, el modelo establece la ruta de la orden con sus dos inspecciones de rigor y la probabilidad final de aceptación de un lote. Los parámetros más importantes del modelo en cuanto a variables y atributos se especifican a continuación:

\section{Las entidades del sistema}

- Las entidades son concebidas como las ordenes de producción.

\section{Atributos}

- Tamaño del lote (TL): que es de 5000 piezas.

- Tiempos de procesamiento $\left(\mathrm{TP}_{(\mathrm{i})}\right)$ : que son los tiempos determinados de procesamiento de cada proceso y que se establece su función densidad de probabilidad.

- Tiempo de terminación (TTO): tiempo en que se completa una orden de producción.

\section{Variables}

- Total de errores (TE): por tipo, número de piezas aceptadas al final del proceso del lote.

- Total de unidades rechazadas (TUR): la constituyen la sumatoria de los puntos de inspección donde se rechazan unidades.

- Probabilidad de Error Humano ${ }_{(\mathrm{i})}(\mathrm{PEH})$ : definida como la probabilidad de error de cada actividad en cada paso del flujo.

- Nivel del error humano (NIEH): establecido como el tiempo de producción adicional por la presencia del error humano en el proceso:

$$
=\sum \text { Tiempo de proceso }(i) \text { con error humano - tiempo de proceso }(i) \sin \text { error }
$$

\section{Resultados y discusión}

Se realizó el diagrama de operaciones y flujo resumiéndose en un infograma, ver figura 1 , donde se muestran las principales tareas del proceso de torno primario. Podemos observar en la figura 1 como se muestran las dos líneas de tiempo de trabajo tanto del trabajador como de la máquina en forma paralela. La mayor cantidad de actividades en número está asociada a los trabajadores, donde ocurren los errores que demoran el proceso y llevan a clasificar una pieza como defectuosa. La nueva tendencia de análisis establece que el trabajador no llega 
a la planta a realizar errores en forma deliberada, sino que el proceso mismo y la falta de un buen sistema de ayuda es quien provoca los errores, Dekker, [19], es por esto que el análisis se enfoca en la prevención del error.

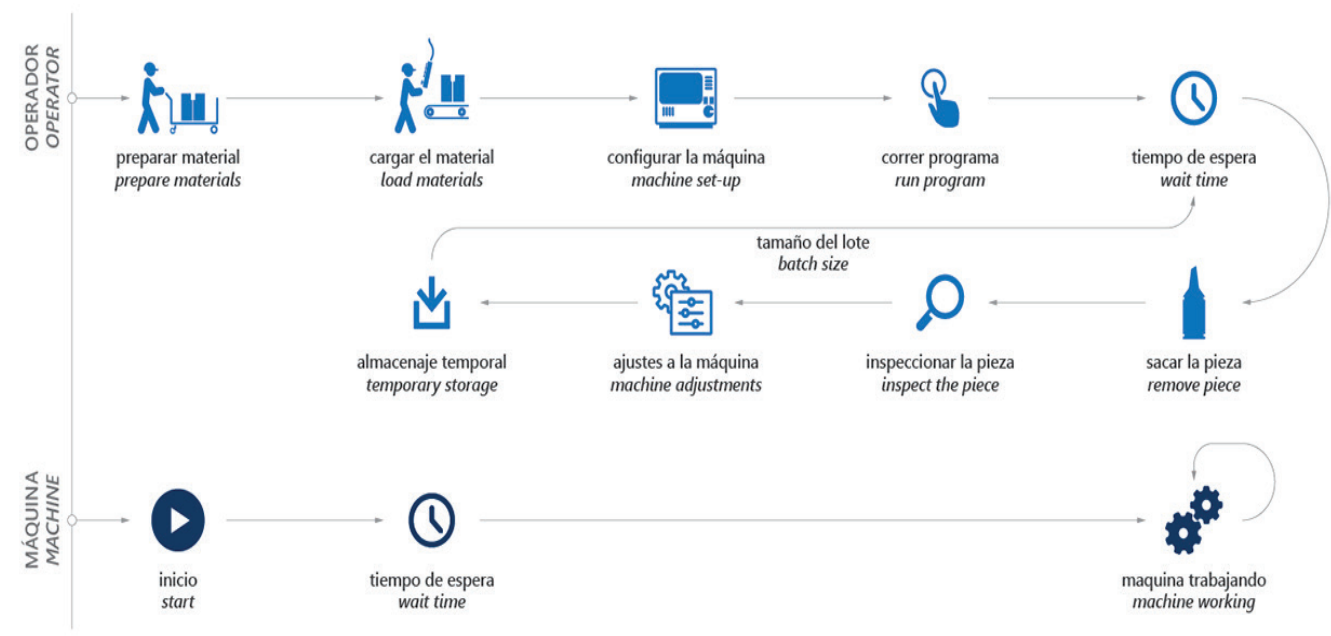

Figura 1. Infograma de proceso de torno primario.

Para un lote de 5000 unidades del total de tiempo promedio de fabricación se consumen un $39 \%$ en labores de preparación, carga de material e inspección las cuales son desarrolladas por el operario. Las pruebas de bondad de ajuste se realizan para un nivel de significancia de 0.05, los valores obtenidos muestran que los tiempos en las diferentes operaciones tienen un comportamiento normal como se muestra en el cuadro 1.

Cuadro 1. Distribución de probabilidad de tiempos de proceso.

\begin{tabular}{|c|c|c|c|}
\hline Tarea & Distribución & Parámetros (segundos) & Valor-p \\
\hline Preparar material & Normal & $14,2.3$ & 0.1561 \\
\hline Cargar material & Normal & $28,5.6$ & 0.0530 \\
\hline Set-up de maquina & Normal & $900,48.2$ & 0.0671 \\
\hline Preparar y correr programa & Normal & 348,20 & 0.1245 \\
\hline Fabricación & Normal & 5,1 & 0.4359 \\
\hline Inspección & Normal & $3,0.5$ & 0.1020 \\
\hline
\end{tabular}

Las primeras cuatro tareas enlistadas en la tabla anterior: preparar material, cargar material, set-up de la máquina y preparar y correr el programa se realizan una única vez por lote de producción mientras que en las dos últimas su tiempo total depende del número de piezas por lote.

Con base a la grabación y diagramación del proceso, se identificaron los errores relacionados a las labores propias de los operarios. Se identificaron un total de 22 errores distribuidos en el área de torno tanto primario como secundario, de los cuales 6 están asociados directamente a torno primario. El $67 \%$ de estos errores en el torno primario se clasifican como errores de acciones y el resto de omisión. En los errores de acción el más probable de acuerdo a la probabilidad de ocurrencia (THERP) es el error al verificar las medidas de las piezas con una probabilidad de 0.2, el cual se da por una mala interpretación de los planos, cansancio del operario o mal uso del set-up de la máquina, con una probabilidad de 0.1 de ocurrencia. Este tipo de error se clasifica como selección en la categoría acción. El set-up es considerado una de las etapas más delicadas y de mayor atención para el operario en la fabricación con 
tecnologías CNC. Esta fase requiere no solo de su habilidad física sino también mental. El error de ingreso del programa es uno de los más bajos con una probabilidad de 0.003, este tipo de error está relacionado con el uso del panel de control del torno donde la distribución del teclado es importante que sea estandarizado, también el tiempo de respuesta del sistema ya que se presiona varias veces una misma tecla en espera de la acción del mismo. Xiaoli y otros [20].

En los errores de omisión se encuentra el error de no verificar el cumplimiento de especificaciones en el lote, dejando correr la producción sin la supervisión del caso. Este error tiene una probabilidad del 0.05 de ocurrencia. Estos errores también pueden ser asociados a la falta de conocimiento o de formación en el personal, el cuadro 2 muestra esto errores. De los trabajadores en el proceso la mayoría son no técnicos en control numérico o uso de torno automatizados. El total de errores identificados se encuentran en el cuadro 2.

Cuadro 2. Total de errores humanos en proceso de torno.

\begin{tabular}{|c|c|c|c|}
\hline Ítem & Área de tornos & PEH & Clasificación \\
\hline 1 & Error al ingresar el programa al torno. & 0.003 & Acción \\
\hline 2 & Error en el set-up de la maquina. & 0.1 & Acción \\
\hline 3 & No activar el baño refrigerante. & 0.05 & Omisión \\
\hline 4 & Cuchilla mal afilada. & 0.001 & Acción \\
\hline 5 & Error al verificar las medidas de las piezas. & 0.2 & Acción \\
\hline 6 & No verificar que la producción del lote siga cumpliendo \\
& con las especificaciones. & 0.05 & Omisión \\
\hline
\end{tabular}

Se corren un total de 254 réplicas para un nivel de confianza del 95\% y un error de 30 segundos en la estimación. El diagrama lógico de la simulación, ver figura 2, muestra las diferentes etapas de producción y las decisiones relacionadas a las variables aleatorias y atributos del lote de producción, así como los errores humanos identificados con sus probabilidades.

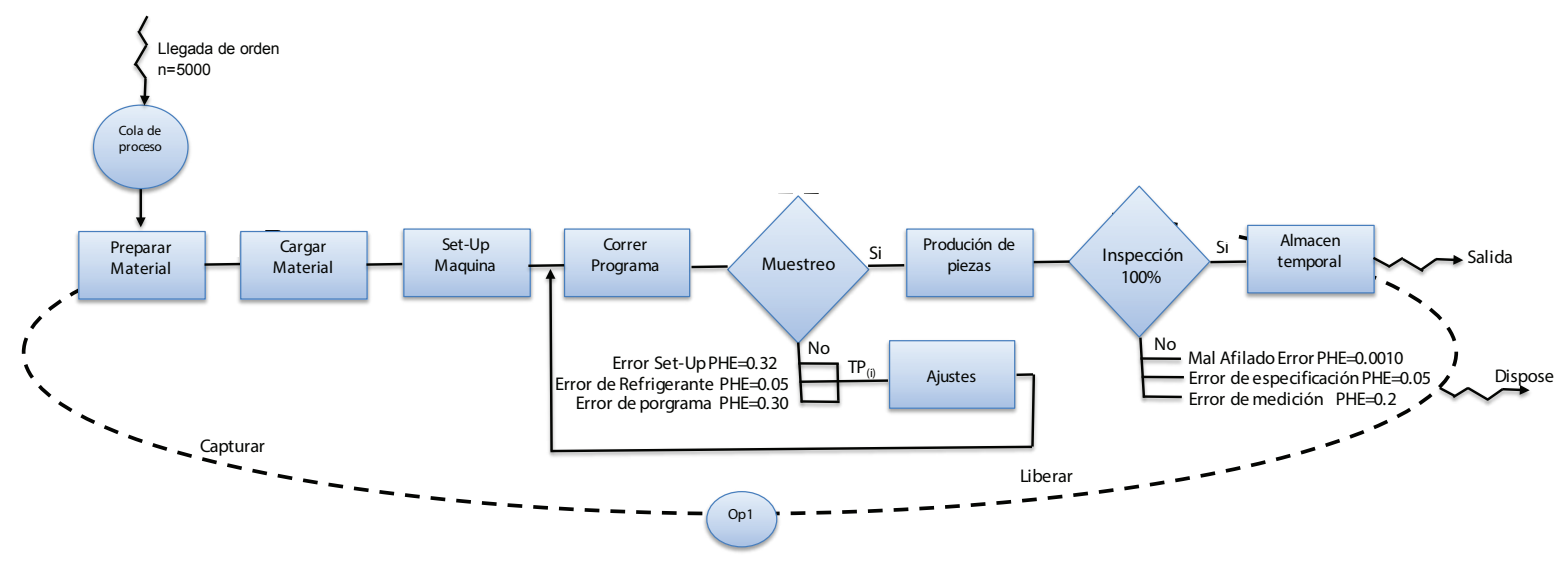

Figura 2. Diagrama de actividades de simulación.

Es importante mencionar que los tres primeros errores: set up, activación del refrigerante y errores de entrada en la digitación del programa son errores que son previsibles y pueden ser corregidos antes de que sea terminado el lote de producción. Se pueden considerar fijos al lote de producción con una repetición baja, ya que una vez corregido no aparece en el resto del lote. En promedio en las 10 primeras piezas estos errores son detectados y corregidos, mientras que el resto de errores asignables después del proceso: error en la medición, error en afilamiento de cuchilla y el error de utilizar especificaciones incorrectas, si hacen o convierten 
a las piezas en defectuosas por lo que la prevención y control deben ser más estrictos. Estos últimos tipos de error se relacionan con acciones repetitivas y por ende fatiga en los operarios ya que se pueden presentar tantas veces como piezas tenga el lote. Estudios previos han detectado que casi el 49\% de la variación en el error humano puede ser justificado por estos factores: fatiga y repetición. Jian, y otros,[21]. En la figura 2 se muestra el diagrama lógico con la representación del modelo simulado.

La validación del modelo se realiza por medio de criterio de experto, donde participaron los ingenieros y encargados del proceso productivo de la empresa. El parámetro de validación fue el tiempo total de una orden de producción de 5000 unidades, calculado por medio de la simulación de la situación actual (Simulación con error humano SEH) este tiempo es de 11.15 horas con una desviación de 0.055.

Para el total de réplicas simuladas en el sistema actual se tiene un promedio de tiempo de terminación (TTO) del proceso considerando todos los errores humanos (CEH) de 40167.0 segundos y una desviación estándar del 201.44, mientras que en el escenario con la no presencia del error humano en la etapa inicial únicamente (SEP) se tiene un TTO de 40003.0 segundos y una desviación de 93.65. El valor del estadístico t para la prueba de medias de estos tiempos es de $\mathrm{p}$-value $=>0.05$ por lo que se rechaza la hipótesis nula, y por lo tanto los tiempos son significativamente diferentes para un 0.05 de significancia. Por otro lado, tenemos que la prueba de medias para el número de unidades rechazadas en estos dos escenarios resulta no ser significativamente diferente para un $p$-value $>0.05$. En este caso el error humano no incrementa el número de defectuosas, pero si el tiempo de terminación el cual se traduce en un mayor costo de producción por unidad. Esta información se complementa con el escenario donde se eliminan los errores al final del proceso (SED). La prueba de medias del tiempo de terminación (CEH/SED) muestra que no existe diferencia significativa entre el tiempo de terminación del lote. Si se analiza el flujo lógico de la simulación esto es esperable ya que estos errores no tienen tiempo de corrección y su presencia únicamente convierte a las piezas en defectuosas. En el caso del número de piezas rechazadas si se encuentran diferencias significativas para un valor de $\mathrm{p}$-value $<=0.05$ en la prueba t de medias, ver tabla 3.

Con el análisis realizado se concluye que el primer grupo de errores impactan el tiempo de producción mientras que los errores al final del proceso impactan la cantidad de piezas aceptadas. El cuadro 3 muestra el resumen de los estadísticos y las pruebas estadísticas realizadas.

Cuadro 3. Resumen de estadísticos y comparación de medias de escenarios.

\begin{tabular}{|c|c|c|c|c|c|}
\hline $\begin{array}{l}\text { Criterio de } \\
\text { evaluación }\end{array}$ & Hipótesis nula & Código & $\begin{array}{l}\text { Media y } \\
\text { desviación } \\
\text { estándar }\end{array}$ & Valor-p & $\begin{array}{c}\text { Resultado } \\
\text { del test }\end{array}$ \\
\hline \multirow{2}{*}{$\begin{array}{l}\text { Tiempo de } \\
\text { terminación TTO }\end{array}$} & \multirow[t]{2}{*}{$H_{0}: T T O_{C E H}=T T O_{S E P}$} & $\mathrm{CEH}$ & $(40167,201.44)$ & \multirow{2}{*}{$\begin{array}{c}\text { Valor-p } \leq \\
0.05\end{array}$} & \multirow[t]{2}{*}{ Rechazado } \\
\hline & & SEP & $(40003,93.65)$ & & \\
\hline \multirow{2}{*}{$\begin{array}{l}\text { Total de unidades } \\
\text { rechazadas TUR }\end{array}$} & \multirow[t]{2}{*}{$H_{0}: T_{C E H}=T_{\text {SER }}$} & $\mathrm{CEH}$ & $(1201.7,30.366)$ & \multirow{2}{*}{$\begin{array}{c}\text { Valor-p > } \\
0.05\end{array}$} & \multirow{2}{*}{$\begin{array}{l}\text { No } \\
\text { rechazado }\end{array}$} \\
\hline & & SEP & $(1202.2,30.44)$ & & \\
\hline \multirow{2}{*}{$\begin{array}{l}\text { Tiempo de } \\
\text { terminación TTO }\end{array}$} & \multirow[t]{2}{*}{$\mathrm{H}_{\mathrm{o}}: \mathrm{TTO}_{\mathrm{CEH}}=\mathrm{TTO}_{\mathrm{SED}}$} & $\mathrm{CEH}$ & $(40172,202.31)$ & \multirow{2}{*}{$\begin{array}{l}\text { Valor-p > } \\
0.05\end{array}$} & \multirow{2}{*}{$\begin{array}{c}\text { No } \\
\text { rechazado }\end{array}$} \\
\hline & & SED & $(40167,201.44)$ & & \\
\hline \multirow{2}{*}{$\begin{array}{l}\text { Total de unidades } \\
\text { rechazadas TTA }\end{array}$} & \multirow[t]{2}{*}{$H_{0}: T T A_{C E H}=T T A_{\text {SED }}$} & $\mathrm{CEH}$ & $(1201.7,30.36)$ & \multirow{2}{*}{$\begin{array}{c}\text { Valor-p } \leq \\
0.05\end{array}$} & \multirow[t]{2}{*}{ Rechazado } \\
\hline & & SED & $(4.41,1.23)$ & & \\
\hline
\end{tabular}

CEH: con error humano.

SEP: sin errores previsibles al inicio del proceso.

SED: sin errores que ocasiona piezas defectuosas al final del proceso.

SEH: sin error humano. 
La simulación también muestra el número de errores humanos esperados para un lote de 5000 unidades, el error en las medidas o en el proceso de medición tiene una frecuencia de 929, este error representa el $80 \%$ del total de errores humanos. Es un error asociado a una acción sumamente repetitiva de mucha concentración para la lectura del instrumento (micrómetro) y del uso del mismo, por lo que la capacitación en la adecuada lectura y la manipulación ergonómicamente correcta del instrumento es importante.

En el caso del error humano en el set-up a pesar que su frecuencia es la más baja si es importante en cuanto al tiempo de ajuste que este representa. El tiempo del set-up esta en promedio en 1800 segundos, representando un 4\% del tiempo total de terminación (TTO) del lote. En la tabla siguiente se muestran los diferentes errores obtenidos en la simulación. Este error se ve directamente relacionado con la experiencia del operario y su formación técnica, requiere de mayor habilidad mental incluyendo el ajuste del programa de control numérico (CNC). Existe muy poca documentación de cómo se efectúan estos procesos y en la empresa apelan a la experiencia de sus colaboradores por realizar esta labor casi de memoria y a su vez transmitir esa información a otros compañeros sin ningún tipo de respaldo de documentación, por lo que la estandarización y documentación del proceso es un medio para disminuir este error.

El error de especificación tiene una frecuencia de 183, ver cuadro 4, el cual tiene su causa fundamental en que los dibujantes carecen de conocimiento sobre normas de dibujo, como por ejemplo ISO, DIN, ASME. Lo anterior se debe a que los dibujantes generan sus planos con exceso de cotas que posteriormente son enviados al operario del proceso de torno con exceso de información que causa confusión en la lectura de las especificaciones.

Cuadro 4. Errores humanos simulados en el proceso.

\begin{tabular}{|c|c|}
\hline Tipo de Error & Cantidad \\
\hline Set up & 1 \\
\hline Medidas & 929 \\
\hline Especificación & 183 \\
\hline Medidas y Especificación & 45 \\
\hline Cuchilla sin filo & 1 \\
\hline
\end{tabular}

Finalmente se reporta el Nivel de error humano (NIEH) con un valor promedio de 1399.0 segundos, equivalente al 3\% del tiempo total de terminación (TTO).

\section{Conclusiones y recomendaciones}

Lo importante para cada empresa es lograr romper la cadena de acciones que pueden llevar al error relacionado con los operarios. Los errores humanos dentro de los procesos productivos relacionados con alta tecnología de control numérico pueden llegar a ser minimizados con la estrategia correcta dependiendo del momento que se presentan en el proceso.

Las labores del set-up de la máquina se pueden considerar como una labor fija al lote, pero de baja frecuencia, y que tiene una probabilidad de error humano de 0.1 que debe ser reforzada por el constante entrenamiento y estandarización del proceso. 
Los errores de especificación y medidas, se encuentran ubicados en la parte final del proceso donde su frecuencia es muy alta aun cuando sus probabilidades son bajas. Las labores donde ocurren estos errores son repetitivas ya que son proporcionales al tamaño del lote. El realizar cambios rotativos de operación con otros operarios y ejercicios de estiramiento son algunas medidas recomendadas para disminuir la fatiga y elevar el nivel de concentración.

El error de especificaciones es sin lugar a duda ocasionado por el exceso de información en los planos y la falta de conocimiento en algunos casos para interpretarlos. De esto es importante que se cuente con:

- Diseño de planos normados

- Diseño de planos divididos por ítem según criticidad

- Diseño de hojas de requerimientos de manufactura por fase en el torno

- Diseño de hojas de requerimientos de insumos en tornos

- Diseño de hojas de control de Proceso o Control de Calidad

El entrenamiento constante y supervisado, así como las herramientas de estandarización de las etapas del proceso de torno son herramientas fundamentales para la disminución del error humano. El error humano es un elemento que influye directamente en el proceso y su disminución puede ocasionar una mejora significativa en el desempeño del mismo.

\section{Referencias}

[1] K. Madhu, S. Babji,, S. Rajagopalan . "Towards predicting human error: Eye gaze analysis for identificationof cognitive steps performed by control room operators. Journal of Loss Prevention in the Process Industries, 42, 35-46, 2016.

[2] D. Wiegmann, S. Shappell, Dicembre, 2015. "Reshaping the way we look at general aviation accidents using the human factors análisis and classification system". Research Gate. Consultado en: https://www.researchgate.net/publication/251800381.

[3] K. Kidam, M. Hurme, MH. Hassim. "Technical analysis of accident in chemical process industry and lessons learnt”. Chem. Eng. Trans. 19, 451-456, 2010.

[4] R. Solis, C. Madriz, "Aplicación de Ergo-Lean Manufacturing en el análisis del valor". Tecnología en marcha, 22:1, 24-28, 2008.

[5] Z. Zang, H. Sharifi. "A methodology for achieving agility in manufacturing organizations". International Journal Operation Production Management, Volumen 20(4), páginas 496-512, 2000.

[6] International Comission of Occupational Heath, Diciembre, 2015. "IEA/ICOH Ergonomics Guidelines for Occupational Health Practice in Industrially Developing Countries". Consultado en: http://www.icohweb.org/ site/news-detail. asp?id=81.

[7] X. Zhang, L. Scmith, R. Schlick, R. Reuth, H. Luczak. " A Human task oriented simulation study in autonomous production cells". International Journal of Production Research. Volumen 1, páginas 1-29, 2007.

[8] M. Sanders, E. McCormick. "Human Factors In Engineering and Design". 7th edición. McGraw-Hill, 1993, USA.

[9] AD. Swain, Guttman, H.E."Handbook of Human Reliability Analysis with Emphasis on Nuclear Power Plant Applications. Albuquerque, Estados Unidos de América: Sandia National Laboratories". (Trabajo preparado para la Comisión Regulatoria Nuclear de Estados Unidos), 1983.

[10] M. Cárdenas. "Fallo humano: la quiebra de un paradigma". Colegio Oficial de Psicología. Universidad de Sevilla. Vol. 27, número 1, págs. 21-51, 2009 Disponible en ,http://www.cop.es/delegaci/andocci//files/contenidos/VOL.\%2027_1_2009/vol.\%2027_1_2.pdf

[11] J. Sureda. Diciembre, 2015. "NTP 620: Fiabilidad humana: evaluación simplificada del error humano Ministerio de Trabajo y Asuntos Sociales España". Disponible en http://www.insht.es/InshtWeb/Contenidos/ Documentacion/FichasTecnicas/NTP/Ficheros/1a700/ntp_620.pdf

[12] Z. Zang, H. Sharifi. "A methodology for achieving agility in manufacturing organizations. International Journal Operation Production Management", Volumen 20(4), páginas 496-512, 2000. 
[13] Jr. Laughery C. Lebiere, S. Archer. "Modelling human performance in complex systems". In Handbook of Human Factors and Ergonomics, edited by G. Salvendy, 3rd edn, pp. 967-996, 2006 (Wiley: New York, NY).

[14] G. Zuelch. "Modeling and Simulation of Human Decision making in Manufacturing Systems". Proceeding del 2006 de la Conferencia de Invierno de Simulación, 2006, páginas 947-953.

[15] S. Robinson, T. Alifantis, J. Edwards. "Knowledge based improvement: Simulation and Artificial intelligence for identifying and improving Human Decision-Making in an Operations System". Journal of the Operational Research Society. 8, 912-921, 2005.

[16] D. Zee. "Modeling decision making and control in manufacturing simulation". International Journal of Production Economics. 100, 155-167, 2006.

[17] X. Zhang, T. Yu, X Xie, D. Zhao. "A comparative Simulation of Organizational strategies in Production Cells Based on Agent Model". Advanced Materials Research. 186, 489-493, 2011.

[18] Instituto Nacional de Seguridad e Higiene en el Trabajo, Diciembre 2015."NTP 620: Fiabilidad humana: evaluación simplificada del error humano (II)" Miisterio de Trabajo y Asuntos Sociañes de españa. Consultado en https://www.insst.es/documents/94886/326775/ntp_620.pdf/20c2aa02-0bb2-4a72-9154-ee2e1d13d024

[19] S. Dekker. " The Field Guide to Understanding "human Error". Third Edition, Ashgate Publishing Limited. Griffith University Australia, 2004.

[20] W. Xiaoli, H. Xin, X. Ruicong,Y. Qingwei. " An Experimental Method Study of User Error Classification in Human-computer Interface. Journal of Software. 8, 2890-2898, 2013.

[21] A. Jian. K. Poh, S. Khong, S. Tee, Y. Wei. " Effects of Stress, Repetition, Fatigue and Work Environment on Human Error in Manufacturing Industries". Journal of Applied Sciences. 14,3464-3471, 2014. 